\title{
Imaging Regulation of Endogenous Gene Expression Using Spliceosome-Mediated Trans-Splicing
}

$\mathbf{R}$ ble increase in our knowledge and understanding of the genetics and molecular biology of human diseases. During the past 2 decades, technologic innovations revolutionized molecular and cell biology. Significant progress in the understanding of the moleculargenetic mechanisms of many diseases has been achieved with the advent of the modern molecular-biologic assays. The development of transgenic animal models of human diseases, which allow the molecular basis of the disease to be studied in a living organism, has provided

\section{See page 1146}

new insights into disease development, progression, and treatment $(1,2)$. In parallel, rapidly developing electronic and computing technology has revolutionized noninvasive imaging, especially optical imaging, nuclear imaging, and MRI. From being the research tools available in only a few centers, PET and MRI are transforming into widely available routine diagnostic procedures.

However, there is a tremendous gap between advances in molecular biology and conventional noninvasive imaging methodologies. The develop-

Received Mar. 15, 2008; revision accepted Mar. 22, 2008.

For correspondence or reprints contact: Vladimir Ponomarev, Molecular Imaging Laboratory, Department of Radiology, Memorial Sloan-Kettering Cancer Center, 1275 York Ave. (Box 501) Z-2063, New York, NY 10021.

E-mail: ponomanv@mskcc.org

COPYRIGHT @ 2008 by the Society of Nuclear Medicine, Inc.

DOI: 10.2967/jnumed.107.049270 ment of unique tracers for direct imaging of every cellular protein and biochemical process of interest is complicated by a vast diversity of potential targets on one hand and the complexity of tracer development and production on the other. A universal approach, such as reporter gene imaging, allows for reliable noninvasive visualization of gene expression and of the activity of different signal transduction pathways through various imaging techniques (3). In this issue of The Journal of Nuclear Medicine, Walls et al. (4) report on the development of the original universal approach for indirect assessment of endogenous gene expression by quantitation of transcribed premessenger RNA (mRNA) molecules using noninvasive genetic reporter imaging.

Transcription and expression levels of genes are typically monitored in vitro by real-time reverse-transcriptase polymerase chain reaction analysis, RNA protection assay, and Northern and Western blotting (5). Reporter assays replace tedious blotting procedures and are widely used because of their convenience, sensitivity, and ability to generate reliable in vitro and in vivo data. The reporter gene usually encodes an enzyme, a receptor, a transporter, or a fluorescent protein, all of which can be easily and cost-effectively monitored. The established methods for noninvasive imaging of reporter gene expression can be effectively introduced into the existing reporter genebased molecular-biologic assay systems (6). In such assay systems, reporter gene expression is linked to an endogenous molecular-genetic process of interest, including regulation of endogenous gene expression at the transcrip- tional and posttranscriptional levels, activation of specific signal transduction pathways, and activation of specific transcription factors.

Regulation of expression of different endogenous genes and signal transduction pathways is commonly assessed using the so-called cis-reporter systems. The final step in the activation of many pathways is the binding of an activated transcription factor to specific elements found in the promoters of various cellular genes (also known as enhancers) (5). To determine the degree of activation of a promoter, and thus the expression of the corresponding gene, different vectors have been designed that use a reporter gene in place of the endogenous cellular gene (7). These reporting systems feature a cis-reporting plasmid or a viral vector that contains a reporter gene driven by a basic promoter element joined to direct repeats of cis-acting DNA elements. After a vector expressing a cis-reporter system of interest is transfected into live cells, the cells can be exposed to an exogenous stimulus, treated with a candidate drug, or further transfected to overexpress a new gene. An increase in the reporter protein activity indicates transcriptional activation of the reporter gene and the signaling pathways converging at these cisacting enhancer elements in cellular responses to the exogenous stimulus, drug action, or functions of the gene of interest.

Cis-reporter systems have been widely used to study regulation of various endogenous gene expressions and of signal transduction pathways. Transgenic models, using numerous response elements or complete promoters, represent a widely accepted 
approach to monitoring gene expression in vivo and to screening exogenous factors such as nutrients and pharmaceuticals for modulation of gene expression (6). An original paper by Doubrovin et al. (8) was the first to show that p53-dependent gene expression can be imaged in vivo with PET and ${ }^{124} \mathrm{I}-2^{\prime}$-fluoro-2'-deoxy-1 $\beta$-Darabinofuranosyl-5-iodouracil and by in situ fluorescence. In that study, a fusion dual-reporter gene comprising herpes simplex virus type 1 thymidine kinase (HSV1-tk) and green fluorescent protein was expressed under control of a p53-specific response element. A positive PET signal corresponded to upregulation of genes in the p53 signal transduction pathway (e.g. p21WAF downstream gene) in response to the DNA damage induced by chemotherapy. A similar approach was applied by Green et al. (9) to noninvasively image the process of albumin synthesis in the liver using a genetic-reporter system and PET. In that study, the level of albumin synthesis was noninvasively assessed in a transgenic animal model by examining the level of HSV1-tk reporter gene transcription from the promoter of the albumin gene after manipulation of the dietary protein content. 9-(4- ${ }^{18}$ F-fluoro-3-[hydroxymethyl]butyl)guanine $\left({ }^{18} \mathrm{~F}\right.$-FHBG) was used as a probe for PET of HSV1-tk reporter gene expression. The ${ }^{18} \mathrm{~F}$ FHBG accumulation in the livers of transgenic mice decreased over the course of a low-protein diet and correlated with albumin mRNA, indicating that endogenous gene expression of albumin can be indirectly imaged with PET. Recent advances of imaging endogenous gene expression using $\mathrm{cis}$ acting genetic reporter systems have been comprehensively reviewed by Iyer et al. (10).

It is important to emphasize that the imaging approach described above directly targets only the transcriptional phase of the gene expression process. Gene expression is further controlled at the posttranscriptional levels by such mechanisms as mRNA processing, mRNA turnover, and mRNA translation. An imaging method that could monitor these processes would provide more accurate and reliable information on the expression levels of the gene of interest.

One approach for imaging gene expression at the mRNA level involves radiolabeled antisense oligonucleotides (RASONs). RASONs are complementary to a small segment of mRNAs of interest and could potentially target any specific mRNA sequence (11). In this context, imaging specific mRNAs with RASONs can be considered a more direct approach to imaging molecular-genetic events. Nevertheless, RASON imaging has several serious limitations, including low specificity of localization (low number of target mRNA molecules per cell, poor stability of binding, degradation by H-RNase), high background activity due to a highly nonspecific extra- and intracellular distribution, limited delivery of reporter probe (poor vascular and cell membrane permeability, inability to penetrate blood-brain barrier), and slow clearance (slow washout of nonbound oligonucleotides from intracellular space).

Walls et al. (4) present an original approach for indirect quantitation of transcribed pre-mRNA molecules using reporter gene imaging. Indirect reporter gene imaging of RNA has several important advantages: highly specific localization, intrinsic signal amplification through a unique enzymatic activity of the reporter gene product that results in local trapping and accumulation of the substrate, low background activity due to rapid clearance and low nonspecific activity, and simplified reporter probe delivery (higher vascular and cell membrane permeability).

Indirect reporter gene imaging of RNA was achieved by using a transsplicing approach. Trans-splicing refers to a process whereby an intron of one pre-mRNA interacts with an intron of another pre-mRNA, enhancing the recombination of splice sites between the 2 conventional pre-mRNAs. Spliceosome-mediated RNA transsplicing (SMaRT) between $5^{\prime}$ and $3^{\prime}$ splice sites within the 2 individual pre-
mRNAs results in a new, chimeric product $(12,13)$. SMaRT has numerous applications, depending on the nature of the sequences that are inserted or trans-spliced onto the defined target, including repair of disease-causing mutant genes at the level of RNA splicing for several disorders such as hemophilia (14) or cystic fibrosis (15). The specificity of the trans-splicing reaction is conferred primarily by the binding domain of the pre-trans-splicing molecules, designed to be complementary to intronic sequences in the target of interest (16). Numerous studies describing ribozyme- or spliceosome-mediated RNA trans-splicing have used the repair of LacZ reporter gene to quantify the efficiency of transsplicing in cells (17). Bhaumik et al. (18) recently provided proof of concept of the ability of SMaRT-mediated optical imaging to visualize endogenous gene expression in vivo using a split-reporter-gene system. In that study, monitoring of real-time gene expression in live animals by RNA trans-splicing was limited to the specific sequence of the reporter gene used (Renilla luciferase). The study by Walls et al. (4) expands this concept and illustrates the principles of a generalizable strategy for imaging levels of any pre-mRNA in living subjects. It can be inferred that the coding domain of the pre-trans-splicing molecules can be easily changed to encode a reporter suitable for imaging with other modalities, including clinical PET and MRI, or to produce a protein that acts therapeutically. This study represents an important step toward a universal imaging methodology of an endogenous mRNA target, with potential applications in research and diagnostics. Imaging regulation of endogenous genes in living subjects using noninvasive techniques can provide a better understanding of normal and diseaserelated biologic processes. In the future, with the advent of tissue-targeted delivery vectors that can be administered systemically (e.g., intravenously), noninvasive visualization of endogenous gene expression may be extended to humans by using humanized reporter 
genetic constructs for clinical nuclear imaging and MRI.

\section{Vladimir Ponomarev \\ Memorial Sloan-Kettering Cancer Center New York, New York \\ REFERENCES}

1. Hanahan D, Wagner EF, Palmiter RD. The origins of oncomice: a history of the first transgenic mice genetically engineered to develop cancer. Genes Dev. 2007;21:2258-2270.

2. Frese KK, Tuveson DA. Maximizing mouse cancer models. Nat Rev Cancer. 2007;7:645-658.

3. Doubrovin M, Serganova I, Mayer-Kuckuk P, et al. Multimodality in vivo molecular-genetic imaging. Bioconjug Chem. 2004;15:1376-1388.

4. Walls ZF, Puttaraju M, Temple GF, Gambhir, SS. A generalizable strategy for imaging pre-mRNA levels in living subjects using spliceosome-mediated RNA trans-splicing. J Nucl Med. 2008;49:1146-1154.

5. Blackwell TK, Walker AK. Transcription mechanisms. WormBook. 2006;September 5:1-16.
6. Albanese C, Hulit J, Sakamaki T, et al. Recent advances in inducible expression in transgenic mice. Semin Cell Dev Biol. 2002;13:129-141.

7. Arnone MI, Dmochowski IJ, Gache C. Using reporter genes to study cis-regulatory elements. Methods Cell Biol. 2004;74:621-652.

8. Doubrovin M, Ponomarev V, Beresten $\mathrm{T}$, et al Imaging transcriptional regulation of p53-dependent genes with positron emission tomography in vivo. Proc Natl Acad Sci USA. 2001;98:93009305.

9. Green L, Yap C, Nguyen $\mathrm{N}$, et al. Indirect monitoring of endogenous gene expression by positron emission tomography (PET) imaging of reporter gene expression in transgenic mice. $\mathrm{Mol}$ Imaging Biol. 2002;4:71-81.

10. Iyer M, Sato M, Johnson M, et al. Applications of molecular imaging in cancer gene therapy. Curr Gene Ther. 2005;5:607-618.

11. Gauchez AS, Du Moulinet D'Hardemare A, Lunardi J, Vuillez JP, Fagret D. Potential use of radiolabeled antisense oligonucleotides in oncology. Anticancer Res. 1999;19:4989-4997.

12. Yang Y, Walsh CE. Spliceosome-mediated RNA trans-splicing. Mol Ther. 2005;12:1006-1012.
13. Pergolizzi RG, Crystal RG. Genetic medicine at the RNA level: modifications of the genetic repertoire for therapeutic purposes by pre-mRNA trans-splicing. C R Biol. 2004;327:695-709.

14. Chao H, Mansfield SG, Bartel RC, et al. Phenotype correction of hemophilia A mice by spliceosomemediated RNA trans-splicing. Nat Med. 2003;9: 1015-1019.

15. Liu X, Jiang Q, Mansfield SG, et al. Partial correction of endogenous DeltaF508 CFTR in human cystic fibrosis airway epithelia by spliceosome-mediated RNA trans-splicing. Nat Biotechnol. 2002;20:47-52.

16. Puttaraju M, DiPasquale J, Baker CC, Mitchell LG, Garcia-Blanco MA. Messenger RNA repair and restoration of protein function by spliceosome-mediated RNA trans-splicing. Mol Ther. 2001;4:105-114.

17. Dallinger G, Puttaraju M, Mitchell LG, et al. Development of spliceosome-mediated RNA transsplicing (SMaRT) for the correction of inherited skin diseases. Exp Dermatol. 2003;12:37-46.

18. Bhaumik S, Walls Z, Puttaraju M, et al. Molecular imaging of gene expression in living subjects by spliceosome-mediated RNA trans-splicing. Proc Natl Acad Sci USA. 2004;101:8693-8698. 